\title{
Stage II Uveal Melanoma AJCC v7
}

National Cancer Institute

\section{Source}

National Cancer Institute. Stage II Uveal Melanoma A/CC V7. NCI Thesaurus. Code C5578.

Stage II includes: IIA (T1 b-d, N0, M0); (T2a, N0, M0); IIB (T2b, N0, M0); (T3a, N0, M0). T1b: Iris: Tumor limited to the iris more than 3 clock hours in size. Ciliary body and choroid: Tumor size category 1 with ciliary body involvement. T1c: Iris: Tumor limited to the iris with secondary glaucoma. Ciliary body and choroid: T umor size category 1 without ciliary body involvement but with extraocular extension less than or equal to 5 $\mathrm{mm}$ in diameter. T1d: Ciliary body and choroid: Tumor size category 1 with ciliary body involvement and extraocular extension less than or equal to $5 \mathrm{~mm}$ in diameter. T2a: Iris: Tumor confluent with or extending into the ciliary body, choroid, or both, with secondary glaucoma. Ciliary body and choroid: Tumor size category 2 without ciliary body involvement and extraocular extension. T2b: Ciliary body and choroid: T umor size category 2 with ciliary body involvement. T3a: Iris: Tumor confluent with or extending into the ciliary body, choroid, or both, with sclera extension and secondary glaucoma. Ciliary body and choroid: T umor size category 3 without ciliary body involvement and extraocular extension. N0: No regional lymph node metastasis. M0: No distant metastasis. (AJCC 7th ed.) 